\title{
Revealing Image Forgery on Digital Images by applying Contrast Enhancement
}

\author{
Sonu John Jacob \\ PG Scholar, Dept. of CSE \\ Amal Jyothi College of \\ Engineering \\ Kerala, India
}

\author{
Krishnalal G. \\ Asst. Prof. Dept. of CSE \\ Amal Jyothi College of \\ Engineering \\ Kerala, India
}

\author{
Jagathy Raj V.P., PhD \\ Proffessor, School of \\ Management Studies \\ CUSAT \\ Kerala, India
}

\begin{abstract}
Image manipulation is a malicious threat that is occurring nowadays. The trustworthiness of images is lost. Malicious users have many photo editing software's to manipulate images in many ways such as brightness changes, contrast manipulations and creating composite images. Here in this, it proposes two novel methods to detect contrast manipulations and composite images. First, it detects the manipulation of images by identifying the image's block level histogram. Second, we propose to identify the manipulation occurring in the images using a high frequency metric method. Once both the methods outputs are obtained it combines the outputs to obtain a final output. Finally it will be able to detect the manipulation that has occurred to the image. Results are summarized at the end and our system is compared with methods used.
\end{abstract}

\section{Keywords}

Image Forgery, Image Manipulation, Brightness, Peak, Gap, Gamma Correction

\section{INTRODUCTION}

The 21 st century has seen the rapid development of technology and making it a digitized environment. Unlike other sources, digital cameras have become the primary source of image capturing. Digital images have become so prevalent in our society. There are different sources from which digital images could be captured. The main threat that is occurring is how much authentic the images are. To manipulate an image is easy but to detect it lays the goal. There are different methods for detecting the manipulation but as such there isn't any universal method. Image manipulations are usually classified as content-changing and content preserving. The content changing forensic methods focus on detecting image tampering such as copy-move [1], splicing and composition[2]-[4] by which the image content is reshaped. Whereas in the content preserving technique the common manipulations such as blur, compression [5]-[6], contrast enhancements [7]-[8] are detected. One example of this content manipulation is the gamma correction technique which is most commonly used method for contrast manipulation.

Different techniques have been adopted to counteract the issues that revolve around image forgery detection. Here basically deal with the histogram of the altered image and check the peak gap relationship. As when contrast enhancement is done the histogram of the altered image will vary along with the peak and gap. Here we find the contrast enhancement for images and also a technique for detecting the source enhanced composite images. In this two methods are used for detecting the manipulated images. Peak - Gap feature of image [9] and High Frequency Energy Metric [10].
Each method has its own output and a combined output is obtained to get the final result. The first method deals with the peak gap relationship by obtaining the block-wise histogram of the image, whereas the second method deals with a high frequency metric which is done by obtaining the histogram of the image. Both the results are combined and a final result is attained. Enough graphs are shown to explain the analysis and accuracy obtained.

The paper is classified as follows. Section 2 deals with the related work, Section 3 describes about the proposed work, In Section 4 has the experimental results and finally Section 5 ends up with the conclusion.

\section{RELATED WORK}

Contrast manipulations have been extensively used to check the authenticity of images. Several techniques have been used for this. Fan proposed a technique for manipulation detection [11] with the aid of EXIF metadata in digital images. They chose the parameters, aperture, shutter speed and ISO speed rating to check the authenticity of images. Stamm globally detected multiple forms of contrast operation with good performance achieved using a histogram of pixel value mapping [12]. The model was further applied to localize contrast enhanced image regions. Gamma correction is another type of contrast manipulation. Ronrong et al. identified how histograms vary when gamma correction is done [13]. Usually in the work, the types of manipulation detected are either contrast or brightness. Gang et al. determined image composition using the object based color consistency check. This was done by feeding the inputs to SVM classifier [14]. The outputs obtained here was less, and could be increased by changing the classifier to neural networks. Zhen et al. [15] introduced a technique for image splicing detection using color edge inconsistency. As such there isn't any detection scheme to detect the contrast manipulations and composite image.

Our method deals in identifying the peak and gaps incurred in the image and identifying a high frequency metric for the image and combining the outputs to attain a final output. Here in our method it can detect both contrast manipulation and composite image. The experiment was done on Matlab2009 and sufficient results were obtained.

\section{PROPOSED METHOD}

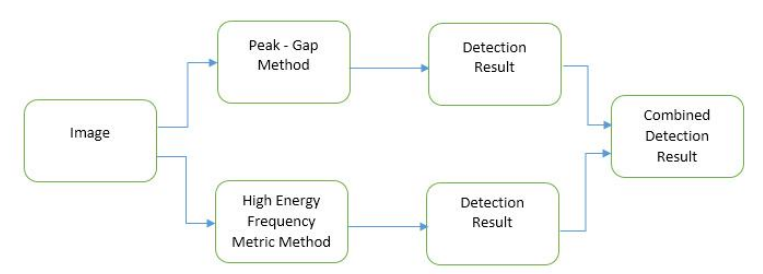

Fig 1: Proposed Technique 
The proposed system is implemented in two methods and a combined result is attained from it to have the final result. The main agenda here is to identify whether the image has undergone any type of manipulations viz contrast manipulation or composite image manipulation.

\subsection{Peak - Gap Method}

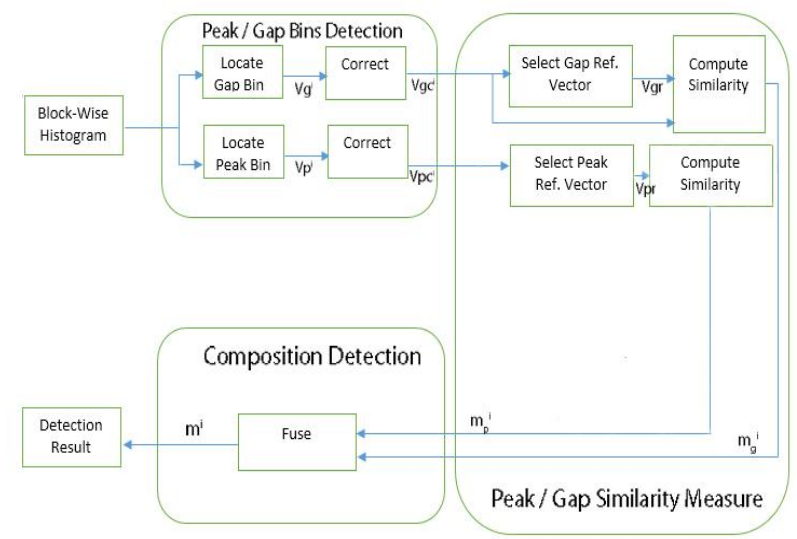

Fig 2: Peak Gap Technique

\subsubsection{Gap Bins Detection}

The peak and gap bins are seen in the histogram of an image. When manipulations like contrast enhancement, brightness increment/decrements are done the peak and gap values changes from the original values. Here in this method the image I is taken and its green color channel is extracted. Once this is done the block-wise histogram of the image is found out and based on this it is able to locate the peak and gap bins. The gap bins are located by the following equation. Once this is done the number of zero height gap bins is identified.

$$
\left\{\begin{array}{c}
h(k)=0 \\
\min \{h(k-1), h(k+1)\}>\tau \\
\frac{1}{2 \omega 1+1} \sum_{k-\omega 1}^{k+\omega 1} h(x)>\tau
\end{array}\right.
$$

Once the block-wise histogram is obtained, next is to detect the bin at $\mathrm{k}$ as a zero height gap bin if it satisfies the above equation. Here the first sub equation refers to that the current bin is null. The second sub equation shows that it keeps two bins larger than the threshold. The third sub-equation properly depicts how the gap bin is extracted. The position of detected gap bins is defined as $\mathrm{V}_{\mathrm{g}}^{\mathrm{i}}=\mathrm{V}_{\mathrm{g}}^{\mathrm{i}}(0), \mathrm{V}_{\mathrm{g}}{ }^{\mathrm{i}}(1) \ldots \ldots \mathrm{V}_{\mathrm{g}}^{\mathrm{i}}(255)$, where $\mathrm{V}_{\mathrm{g}}^{\mathrm{i}}(\mathrm{k})=1$ indicates a gap bin. $\mathrm{V}_{\mathrm{g}}^{\mathrm{i}}(\mathrm{k})=0$ otherwise.

\subsubsection{Peak Bins Detection}

The peak bins behave as impulsive noise which are usually located by median filtering. The median filter is used to remove the impulsive noise and also the salt and pepper noise. The gap bins are filled with average of neighboring bins, then median filtering is applied to the gap filled histogram. The peak positions are located by thresholding the difference between the gaps filled histogram and its filtered version. The peak positions are detected as $\mathrm{V}_{\mathrm{p}}{ }^{\mathrm{i}}=\mathrm{V}_{\mathrm{p}}{ }^{\mathrm{i}}(0), \mathrm{V}_{\mathrm{p}}{ }^{\mathrm{i}}(1) \ldots . . \mathrm{V}_{\mathrm{p}}{ }^{\mathrm{i}}$ (255), where $\mathrm{V}_{\mathrm{p}}{ }^{\mathrm{i}}(\mathrm{k})=1$ indicates a gap bin. $\mathrm{V}_{\mathrm{p}}{ }^{\mathrm{i}}(\mathrm{k})=0$ otherwise.

The peak-gap bins that are obtained sometimes may not appear as the histogram of the image is too narrow to cover such bin position. In order to overcome this, effective detection range (EDR) is introduced for the $i^{\text {th }}$ block-wise peak/gap positions and is defined as set of gray levels around which the histogram bins are not all zeros.

\subsubsection{Peak/Gap Similarity Measure}

In order to obtain the gap based similarity it is necessary to first set a reference position vector for each of it. Then the block can be classified by the similarity between its position vector and the reference vector. The reference gap position vector for the located region can be set as $\mathrm{V}_{\mathrm{gr}}=\mathrm{V}_{\mathrm{gc}}{ }^{\mathrm{k}}$, where

$$
\mathrm{K}=\arg 1,2, \ldots . \mathrm{Nb} \max \left(\left\|\mathrm{V}_{\mathrm{gc}}{ }^{\mathrm{i}}\right\|\right)
$$

When no gap involved pair occur it is marked as $\mathrm{m}_{\mathrm{g}}^{\mathrm{i}}=-1$ or else as 0 . The gap based similarity is attained using the following equation

$$
m_{g}^{i}=\frac{\Sigma_{k \in \Omega_{i} \cap \Omega_{g r}} V_{g c}^{i}(k) \cdot V_{g r}(k)}{\sum_{k \in \Omega_{i} \cap \Omega_{g r}} V_{g c}^{i}(k) \cdot V_{g r}(k)+\bar{V}_{g c}^{i}(k) \cdot V_{g r}(k)+V_{g c}^{i}(k) \cdot \bar{V}_{g r}(k)}
$$

Once the Gap similarity measure it is needed to obtain the peak similarity measure to fuse both the similarities to detect any kind of manipulation in the image. Most of the image block usually owns a narrow histogram, the gaps bins might be unavailable in not a few blocks/. The narrow histogram without gap bins might carry with peak bins.

The reference peak position vector $\mathrm{V}_{\mathrm{pr}}$ is created by combining the peak position vectors which are available from the source region of $\mathrm{V}_{\mathrm{gr}}$. As mentioned in equation 3 the gap reference vector is computed in the same way using the same equation. When no gap involved pair occur it is marked as $\mathrm{m}_{\mathrm{p}}^{\mathrm{i}}=-1$ or else as 0 .

\subsubsection{Similarity Fusion}

Before fusion the block-wise similarities $\mathrm{m}_{\mathrm{p}}^{\mathrm{i}}=-1$ and $\mathrm{m}_{\mathrm{g}}^{\mathrm{i}}=-1$ are updated. The resulting similarity for the $\mathrm{i}^{\text {th }}$ block is denoted as $\mathrm{m}^{\mathrm{i}}$ can be generated by fusing the peak/gap based similarities. When $\mathrm{m}_{\mathrm{p}}^{\mathrm{i}} \neq-1$ and $\mathrm{m}_{\mathrm{p}}^{\mathrm{i}} \neq-1$ it is computed as

$$
\mathrm{m}^{\mathrm{i}}=\left(\mathrm{m}_{\mathrm{p}}^{\mathrm{i}}+\mathrm{m}_{\mathrm{g}}^{\mathrm{i}}\right) / 2
$$

When $\mathrm{m}_{\mathrm{p}}^{\mathrm{i}}=-1$ and $\mathrm{m}_{\mathrm{g}}^{\mathrm{i}}=-1, \mathrm{~m}^{\mathrm{i}}=\max \left(\mathrm{m}_{\mathrm{p}}^{\mathrm{i}}, \mathrm{m}_{\mathrm{g}}^{\mathrm{i}}\right)$ This is how fusion is done. Using this result it is able to detect the manipulation occurred. The manipulation occurred are checked as contrast manipulations viz gamma correction and $\mathrm{s}$ mapping, and also checks for composite image manipulation. Here it basically checks for two types of composite image detection i.e. single source composite image and double source composite image.

As it performs the manipulation check, it is able to note that the accuracy for this check is very much less. So in-order to overcome this another technique known as high frequency energy metric and then it combines both results to produce a final result.

\subsection{High Frequency Energy Metric}

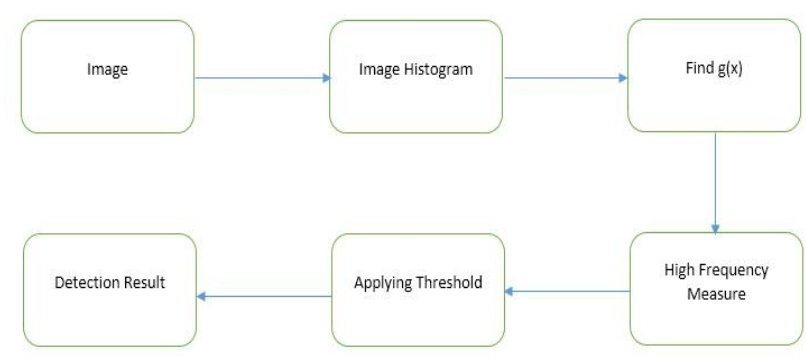

Fig 3: High Frequency Energy Detection Technique 
This is the block diagram for the second method. The peaks and gaps which are introduced to images histogram are contrast enhancement artifacts which is used to perform the manipulation detection. Here this method works by obtaining a weighted measure $\mathrm{F}$ of an image histogram's high frequency component performing a threshold test to determine if contrast enhancement has occurred. There exists a class of naturally occurring unaltered images, which is referred to as high end or low end saturated. High end saturation occurs when an image is captured under bright lighting conditions and it results in a substantial number of pixels taking on a value of 255 , thus creating a sharp peak.

Low end saturation arises in images taken in dark lighting environments and corresponds to an impulsive peak at the pixel value 0 . This is problematic due to the fact that the Fourier transform of an impulse is a constant function, which will in turn lead to a large value of $\mathrm{F}$ and bias the detector towards deciding that contrast enhancement has occurred. To overcome this we pre-multiply $\mathrm{h}(\mathrm{x})$ by a pinch off function $\mathrm{p}(\mathrm{x})$ to obtain $\mathrm{g}(\mathrm{x})$, where $\mathrm{h}(\mathrm{x})$ is the histogram.

$$
g(x)=p(x) \cdot h(x)
$$

$\left\{\begin{array}{cc}\frac{1}{2}-\frac{1}{2} \cos \left(\frac{\pi x}{N p}\right), & \text { if } x \leq N p \\ \frac{1}{2}+\frac{1}{2} \cos \left(\frac{\pi(x-255+N p)}{N p}\right), & \text { if } x \geq 255-N p \\ 1, \text { else } & \end{array}\right.$

Where the width of the pinch off region $\mathrm{Np}$ is typically around 4 pixels. We use $\mathrm{g}(\mathrm{x})$ to calculate the high frequency measure $\mathrm{F}$ according to the formula.

$$
F=(1 / N) \sum_{\omega}|\beta(\omega) \cdot G(\omega)|
$$

Where $N$ is the total number of pixels in the image, $G(\omega)$ is the Fourier transform of $\mathrm{g}(\mathrm{x})$, and $\beta(\omega)$ is a weighting function which takes values between 0 and 1 . The purpose of $\beta(\omega)$ is to de-emphasize low frequency regions of $G(\omega)$. A threshold test is performed to values of $\mathrm{F}$ to determine the manipulation that has occurred. Now once both the methods are attained, we compare the outputs of both the methods to obtain a final output.

\section{EXPERIMENTAL RESULTS}

The images selected to be included in dataset is from the UCID dataset, which consists of images in the TIF format. Here contrast enhancement is enforced on the image to detect it. The contrast enhancement that is enforced are gamma correction and s mapping. Gamma correction is a type of contrast enhancement which used to change the luminous intensities of the image and S-mapping is done to change the middle tone pixels. Once contrast enhancement is done the images are compressed too. The curves chosen for gamma correction is shown below.

$$
\mathrm{m}(\mathrm{x})=\operatorname{round}\left(255(\mathrm{x} / 255)^{\wedge} \mathrm{r}\right.
$$

Where $r$ represents the amount of change. $r$ is chosen as 0.7 and $\mathrm{x}$ represent the current pixel. The second pair of curves is $\mathrm{S}$ mapping. The $\mathrm{S}$-mapping is

$$
\mathrm{m}(\mathrm{x})=\operatorname{round}(255(\arcsin (2 \mathrm{x} / 255-1) / \pi+0.5)
$$

In order to detect single source enhanced composite image, the image itself is manipulated. Single sourced composite image means a part of the image is copied into the same image itself. Whereas a double sourced composite image means a part of an image is copied into another image. Here in this work to create a single sourced composite image we itself copy a part of the image to itself.

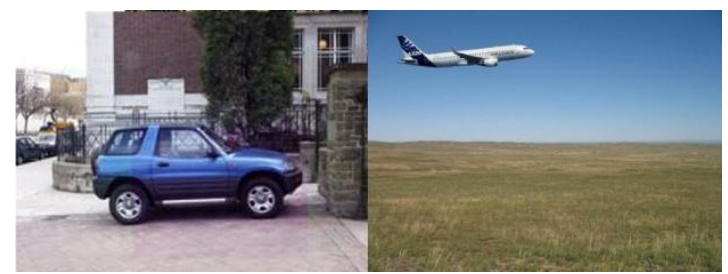

Fig 4: Single and double sourced composite image

The first image shows a single sourced composite image in which we see a small rectangular portion in violet color. The user itself has manipulated it for detection. A part of the image is copied into that region. The second image represents a double sourced composite image in which we see that an aircraft is copied into it. Hence we will be able to detect the kind of manipulation occurred.

Initially we took 70 images and manipulated the images based on the above mentioned curves and generated a total of 280 images including the original images. In addition to it we added 20 double sourced composite image and obtained a total of 300 images and classified each image into its manipulated folders based on its type of manipulation. Based on this the manipulation detection is done and we were able to identify the manipulation that occurred, i.e. single sourced or double sourced composite image or any type of contrast manipulation, i.e. gamma correction or s mapping.

The analysis of the work is done with the help of a correlation matrix which consist of true positive, false positive, true negative, and false negative. In addition to this we find the sensitivity, specificity, positive predictive value, negative predictive value, false positive rate, false negative rate, false detection rate and accuracy.

Fig 5 illustrates the TP, FP, TN, and FN of the images that were taken in account for the manipulation detection scheme.

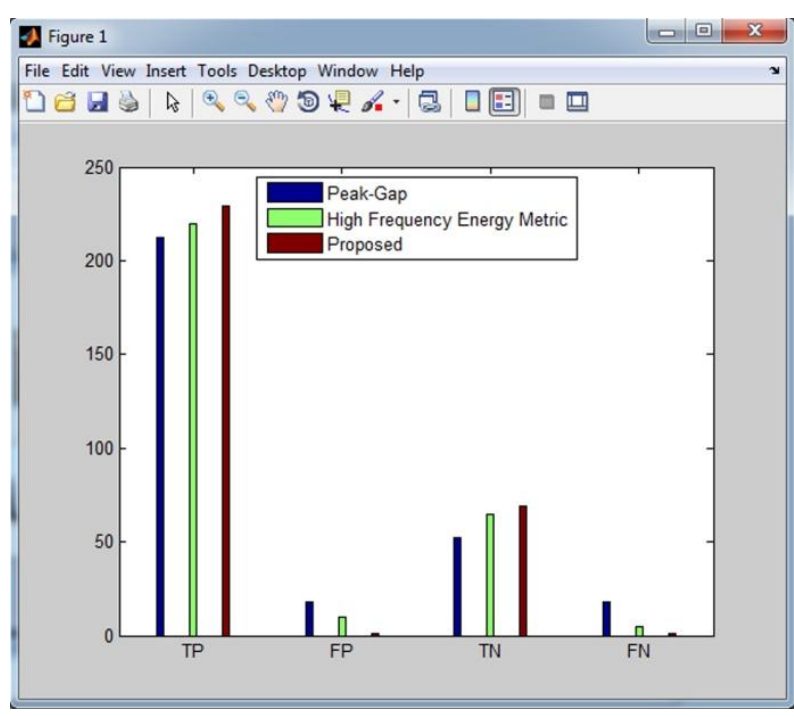

Fig 5: TP, FP, TN, FN

Next the sensitivity, specificity and accuracy is calculated. Sensitivity is also known as recall or true positive rate. All the 3 parameters estimated here attains a value of 1 for the proposed method. 


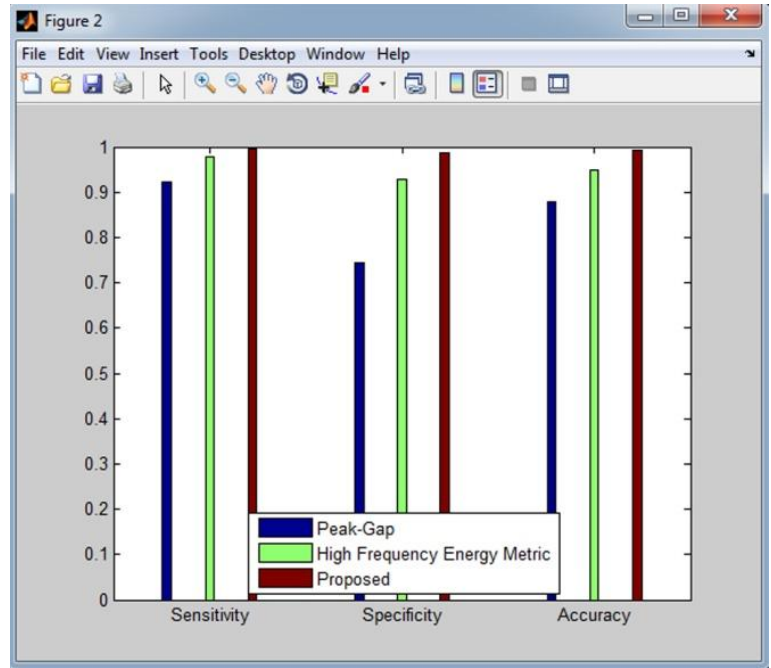

Fig 6: Sensitivity, Specificity, Accuracy

The positive predictive value (precision) and negative predictive value is found. Both parameters calculated here attains a value of 1 for the proposed method.

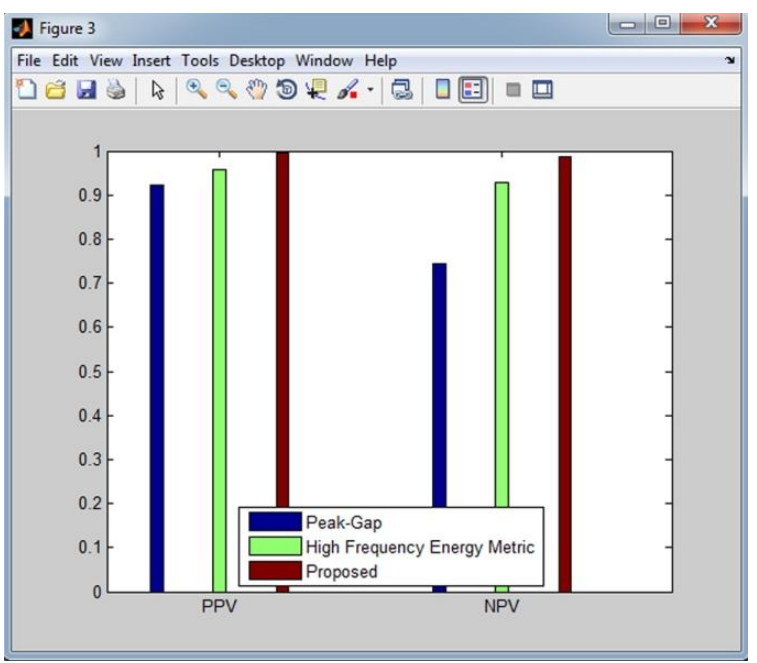

Fig 7: PPV, NPV

Finally the F-SCORE for the three methods is calculated and it shows better value for the proposed method.

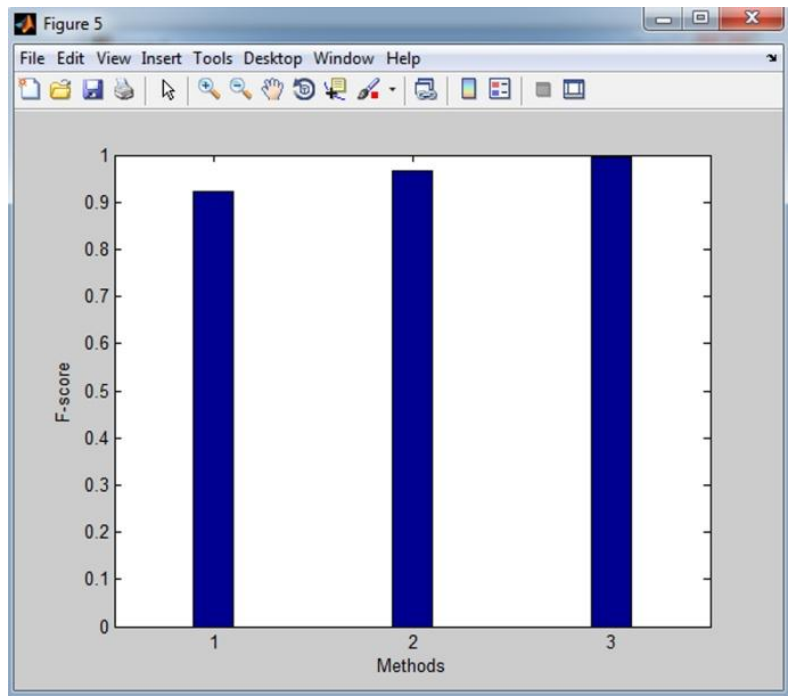

Fig 8: F-score
This is how analysis of the work is done. For the common manipulations to deceive human eyes, our proposed method exhibits excellent performance and consistency in detection and better acccurracy over the other two techniques.

\section{CONCLUSION}

In this we proposed a method to detect the kind of manipulation that has occurred on the image and the work for it was done and it showed. Two methods were used to obtain a final output. Each method produced its own output and identified the manipulation that had occurred. The peaks and gaps extracted from the image dint show as much of accuracy for the detection where as it showed that the high frequency method showed greater accuracy. The manipulation detected here Contrast Enhancement, S- Mapping, single and double sourced composite image. As a future enhancement we can detect brightness manipulation by extracting features and applying it to some classifiers viz SVM, neural networks.

\section{REFERENCES}

[1] S. Bayram, H. T. Sencar and N. Memon, "An Efficient and Robust Method for Detecting Copy-move Forgery," in Intl. Conf on Acoustics, Speech and Signal Processing, Taipei, 2009.

[2] Y.-F. Hsu and S.-F. Chang, "Image Splicing Detection Using Camera Response Function Consistency and Automatic Segmentation," in Intl. Conf. on Multimedia and Expo, Beijing, 2007.

[3] I. Yerushalmy and H.Hel-Or, "Digital Image Forgery Detection Based on lens and sensor abberration," Int J. Comput, Vis, vo 92, no. 1, pp. 71-91, 2011

[4] J.O'Brien and H. Farid "Exposing photo manipulation with inconsistent reflections," ACM Trans. Graph, vol .31 , no. 1 , pp. $1-11,2012$

[5] Z. Fan and R. L. Queiroz, "Identification of Bitmap Compression History: JPEG Detection and Quantizer Estimation," IEEE Trans. on Image Processing, vol. 12, no. 2, pp. 230-235, 2003.

[6] T. Bianchi and A. Piva, "Detection of non - aligned double JPEG compression based on integer periodicty maps, "IEEE Trans. Inf.Forensics Security, vol 7, no.2, pp 842-848, Apr 2012

[7] M.C. Stamm and K.J.R.Liu, "Forensic estimation and reconstruction of a contrast enhancement mapping," in Proc. IEEE Int Conf. Acoust. Speech Signal, Dallas,TX,USA, Mar.2010, pp 1698-1701

[8] P.Ferrara, T. Bianchiy, A. De Rosaz and A.Piya "Reverse Engineering of double compressed images in the presence of contrast enhancement," inProc. IEEE Workshop Multimedia Signal Process, Pula, Croatioa, Sep/Oct. 2013, pp. 141-146.

[9] Gang Cao, Yao Zhao, Rongrong Ni, Xuelong Li, "Contrast Enhancement-Based Forensics in Digital Images", Information Forensics and Security, IEEE Transactions on, on page(s): 515 - 525 Volume: 9, Issue: 3, March 2014 
[10] M. C. Stamm and K. J. R. Liu, 'Blind Forensics of Contrast Enhancement in Digital Images," IEEE Conferecne, Apr. 2008.

[11] J. Fan, H. Cao, and A. C. Kot, lemph\{“Estimating EXIF parameters based on noise features for image manipulation detection,"\} IEEE Trans. Inf. Forensics Security, vol. 8, no. 4, pp. 608-618, Apr. 2013.

[12] M. C. Stamm and K. J. R. Liu, "Forensic detection of image manipulation using statistical intrinsic fingerprints," IEEE Trans. Inf. Forensics Security, vol. 5, no. 3, pp. 492-506, Sep. 2010

[13] Gang Cao, Yao Zhao, Rongrong Ni, "Forensic Estimation in Gamma Correction in Digital Images, "
Proceedings of 2010 IEEE 17th International Conference on Image Processing September 26-29, 2010, Hong Kong

[14] Gang Cao, Yao Zhao, Rongrong $\mathrm{Ni}$, “'Image Composition Detection Using Object-based Color Consistency," IEEE Proceedings of 9th International Conference on Signal Processing, 2008

[15] Zhen Fang, Shuozhong Wang, Xinpeng Zhang, "Image Splicing Detection Using Color Edge Inconsistency," International Conference on Multimedia Information Networking and Security, 2010 